\title{
LETTER OPEN GPR160 is a potential biomarker associated with prostate
}

\section{cancer}

Signal Transduction and Targeted Therapy (2021)6:241

\section{Dear Editor,}

Prostate cancer (PC) is one of the most common noncutaneous cancers among men worldwide with a relatively higher incidence and mortality rate. ${ }^{1}$ Conventional screening for prostate cancer relies on prostate-specific antigen (PSA), a valuable biomarker but has some deficiencies. Radical prostatectomy, androgen ablation, and radiotherapy are still commonly used treatments for localized prostate cancer. Androgen ablation is a procedure aimed at suppressing hormone production or blocking the function of the androgen receptor. At the early stage, prostate cancer growth is dependent on androgen, while most patients will ultimately transform to a hormone-refractory state. New diagnostic tools and therapeutic strategies are thus in high demand to curtail this situation. GPR160 belongs to the class A GPCR subfamily and was deorphanized recently. Its endogenous ligand is CARTp (cocaine- and amphetamine-regulated transcript peptide) responsible for neuropathic pain in rodents. ${ }^{2}$ We have shown previously that GPR160 is highly expressed in prostate cancer tissue samples and its knockdown by lentivirus-mediated short hairpin RNA constructs targeting the human GPR160 gene (ShGPR160) resulted in prostate cancer cell apoptosis and growth arrest both in vitro and in athymic mice. ${ }^{3}$

To investigate the relationship between expression of GPR160 and prostate cancer clinicopathologic characteristics, we examined the expression of GPR160 in prostate samples obtained from 224 patients during prostatectomy by in situ hybridization and immunohistochemistry (Figure S1). Of the tissue samples obtained from 224 patients receiving prostatectomy, 199 and 158 of them were successfully assessed for RNAscope ${ }^{\circledR}$ and immunohistochemistry analyses, respectively (Fig. 1a). Positive readouts for GPR160 expression were $77.4 \%$ (154/199) and 65.8\% (104/158) in mRNA and protein levels, respectively; both were significantly higher in cancerous than that in adjacent normal tissues $(P<$ 0.0001 , Figs. $1 \mathrm{~b}-\mathrm{e})$, demonstrating consistency between the two parameters.

As shown in Table S1, the mean age at diagnosis was 66.85 years, ranging from 51 to 82 years. Positive GPR160 RNA transcription was associated with higher Gleason grading scores (Fisher's exact test, $P=0.026$ ), the later stage of prostate cancer (Chi-square test, $P=0.045$ ) and elevated PSA levels (unpaired $t$ test, $P=0.048$, between strong and moderate expressions, Figs. If-h). Table S2 illustrates the correlation between GPR160 protein levels and the clinical status of enrolled patients with the mean age at diagnosis of 66.84 years (ranging from 47 to 82 years). It was found that moderate GPR160 expression was linked with older ages compared with that of weak and strong expression groups $(P=0.009$ and $P=0.026$, respectively). GPR160 protein expression was also correlated with metastatic status using the $M$ descriptor of the TNM (tumor, node, metastasis) classification system $(P=0.041$, Figs. $1 \mathrm{i}-\mathrm{j})$.

The role of GPR160 in the metastatic potential of prostate cancer cell line 22Rv1 was investigated in parallel with the human

\author{
; https://doi.org/10.1038/s41392-021-00583-7
}

prostate epithelial cell line RWPE-1 and human prostate stromal cell line WPMY-1. We employed the western blot technique to analyze GPR160 and the epithelial-mesenchymal transition (EMT) hallmarks including E-cadherin, $\mathrm{N}$-cadherin, vimentin, snail, fibronectin 1, and ZO-1 in RWPE-1 and WPMY-1 cells following transfection of pCMV6-Entry vector encoding hGPR160. Figures $1 \mathrm{k}$, $1 \mathrm{l}, 1 \mathrm{q}$, and $1 \mathrm{r}$ show that increased GPR160 expression was associated with elevated E-cadherin and ZO-1 but decreased $\mathrm{N}$-cadherin, vimentin, and snail levels in RWPE-1 cells. Figures $1 \mathrm{~s}$ and $1 \mathrm{t}$ show that similar expression deference in WPMY-1 cells. To verify these results, we also detected the EMT hallmarks in GPR160-silenced prostate cancer cells (22Rv1). As displayed in Figs. $1 \mathrm{~m}-\mathrm{p}$, the expression levels of E-cadherin and ZO-1 were decreased, whereas that of $\mathrm{N}$-cadherin, vimentin, snail, and fibronectin 1 increased, suggesting that the GPR160 silenced cells (22Rv1) acquired invasive and metastatic properties, whereas RWPE-1 and WPMY-1 cells over-expressing GPR160 lost these properties. In phenotype assays, increased cell migration in GPR160 silenced cells (22Rv1 and PC3) and decreased cell migration in GPR160 overexpressed cells (RWPE-1 and WPMY-1) were observed (Figs. $1 \mathrm{u}$ and 1v). A similar trend was also noted in invasion assay (Figs. $1 \mathrm{w}$ and $1 \mathrm{x}$ ). At the same time, wound healing assay exhibited more migration after silencing GPR160 in 22Rv1 and PC3 cells but less migration after over-expressing GPR160 in WPMY-1 cells (Fig. 1y).

As the second leading cause of death among men in the United States, poor prognosis is associated with advanced or metastatic prostate cancer. Despite the wide application of PSA in its diagnosis, novel and specific prostate biomarkers would certainly improve our ability to manage this deadly disease. In this study, we explored the relationship between GPR160 expression and the clinical status of prostate cancer. Both mRNA and protein levels of GPR160 were correlated with age, PSA, prostate size, Gleason score, TNM stage, lymph node metastasis, and nerve invasion in all enrolled patients.

Previous studies showed that the transcription level of GPR160 in several prostate cancer cell lines (PC3, LNCaP, DU145, and 22Rv1) was significantly higher and its expression was associated with apoptosis, microtubule cytoskeleton, cytokine activity, cell cycle, mitosis, and programmed cell death. ${ }^{3}$ The present study extended our scope to directly involve tissues from prostatectomy and to analyze GPR160 mRNA and protein levels in the context of clinical relevance. At the same time, both Gleason grade and stage classification are positively correlated with GPR160 mRNA levels (Table S1). This may provide an alternative parameter to predict the degree of malignancy in a semi-quantitative manner.

EMT is an important process during development by which epithelial cells acquire mesenchymal, fibroblast-like properties and show reduced intercellular adhesion and increased motility. ${ }^{4}$ It is implicated to play a critical role in tumor progression and malignant transformation by gaining invasive and metastatic 

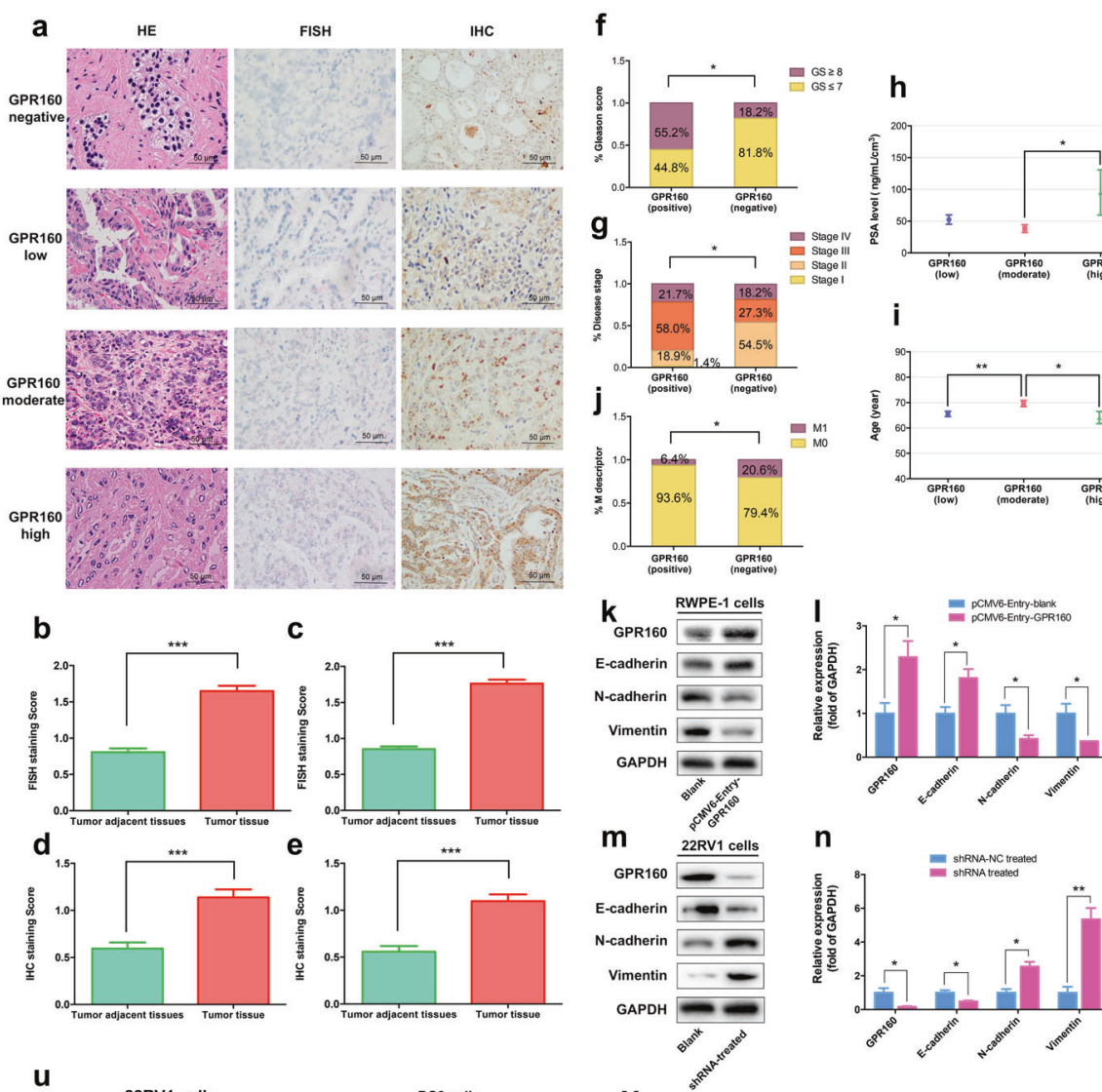

n
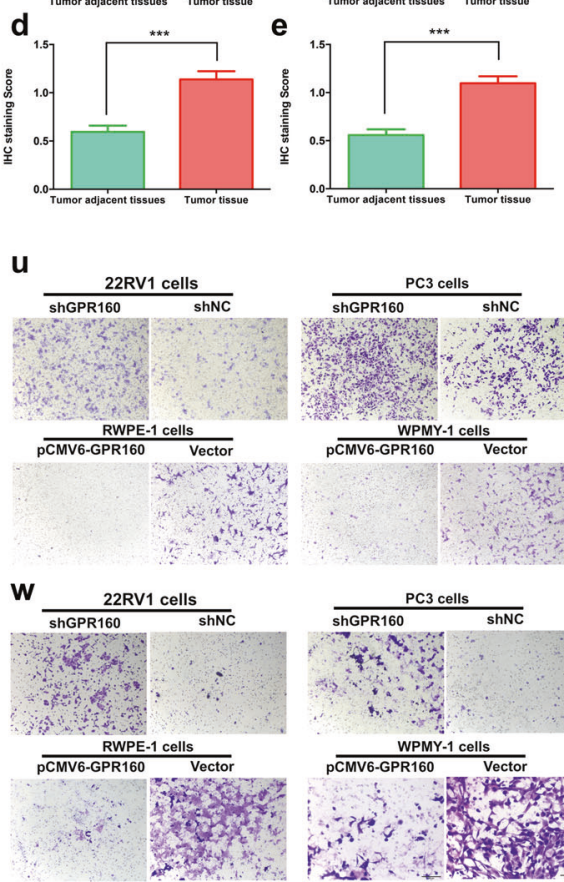

c

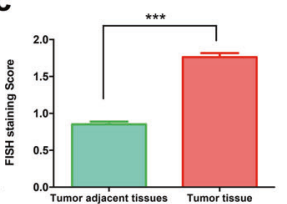

d
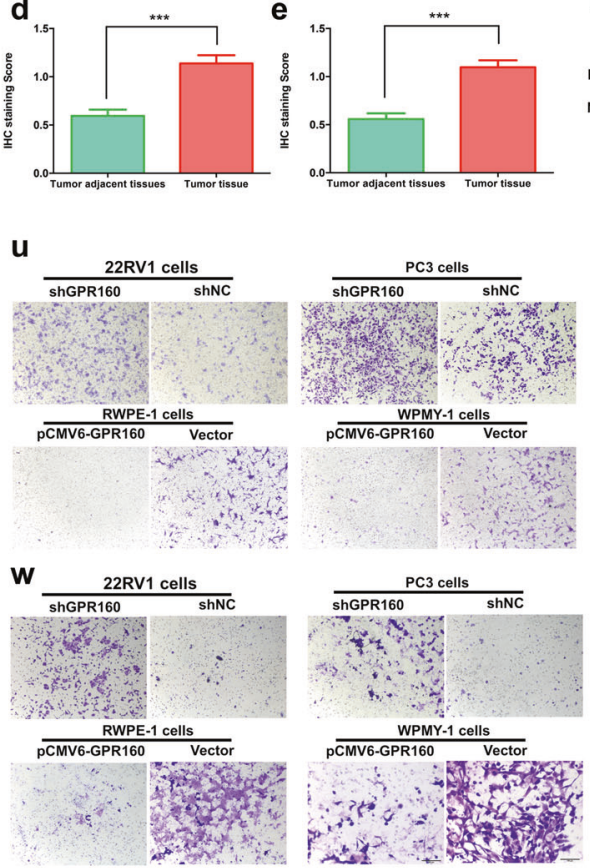

V

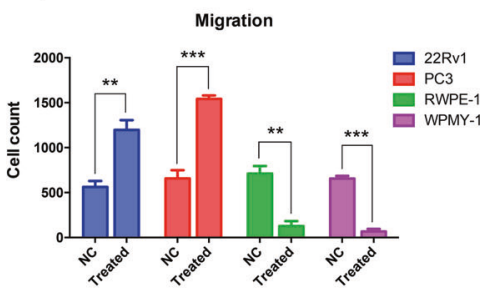

X

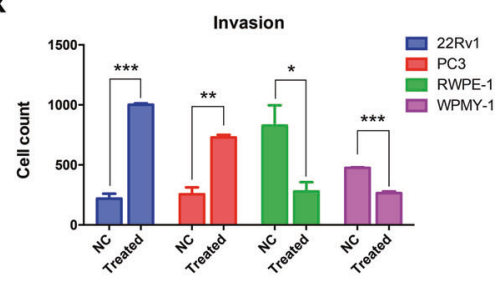

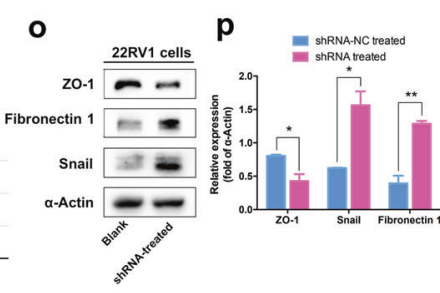
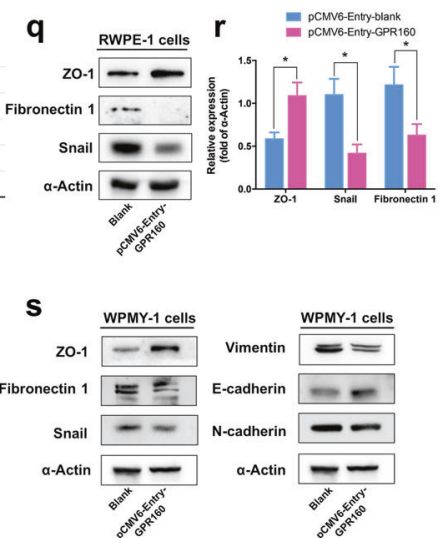

$t$

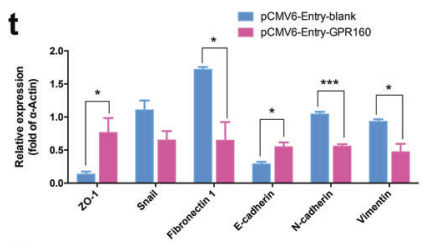

y

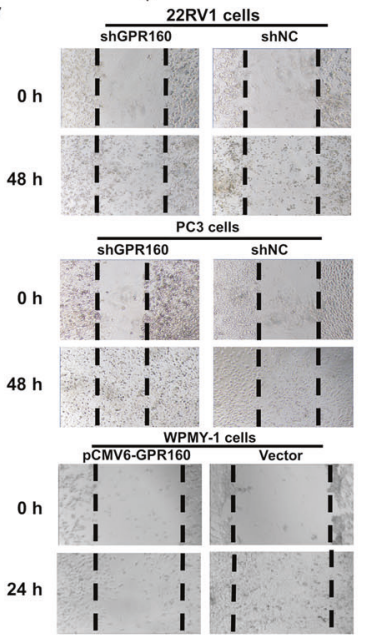

Fig. 1 Higher expression of GPR160 in cancerous human prostate tissues and relationship between GPR160 expression levels and clinical characteristics of enrolled patients. a Images of GPR160 expression detected by RNAscope (mRNA, FISH)) and immunohistochemistry (protein, IHC). HE, hematoxylin and eosin, scale bars, $50 \mu \mathrm{m}$. b 143 samples containing both normal and cancerous tissues used for mRNA analysis with paired $t$ test. c 167 samples containing cancerous and 143 samples containing normal tissues used for mRNA analysis with unpaired $t$ test. d 112 samples containing both normal and cancerous tissues used for immunohistochemistry analysis with paired $t$ test. e 140 samples containing cancerous and 112 samples containing normal tissues used analysis with unpaired $t$ test. Data presented are means \pm SEM of up to three independent reading; ${ }^{* *} P<0.0001$. $\mathbf{f}$ Gleason scores vs. GPR160 mRNA levels in the prostate cancer tissue. $\mathbf{g}$ Disease stage vs. GPR160 mRNA levels in the prostate cancer tissue. h PSA levels in patients with elevated GPR160 mRNA transcription. i Age-related GPR160 protein intensity. $\mathbf{j}$ M scores in patients with or without GPR160 protein expression. Data shown are means \pm SEM; ${ }^{*} P<0.05$ using unpaired $t$ test. k Expression of the epithelial-mesenchymal transition (EMT) hallmarks E-cadherin, N-cadherin, and vimentin as well as GPR160 in blank and GPR160 gene transfected cells. I Quantitative densitometric analysis of k. $\mathbf{m}$ Expression of the three EMT hallmarks and GPR160 in blank and GPR160 gene silenced cells. $\mathbf{n}$ Quantitative densitometric analysis of $\mathbf{m}$. o Expression of the EMT hallmarks ZO-1, snail, and fibronectin 1 in blank and GPR160 gene silenced cells. p Quantitative densitometric analysis of o. q Expression of the EMT hallmarks ZO-1, snail, and fibronectin 1 in blank and GPR160 gene transfected RWPE-1 cells. $\mathbf{r}$ Quantitative densitometric analysis of q. $\mathbf{s}$ Expression of the EMT hallmarks ZO-1, snail, and fibronectin 1 in blank and GPR160 gene transfected WPMY-1 cells. $\mathbf{t}$ Quantitative densitometric analysis of s. u, Migration of GPR160 gene silenced or transfected cells. v Quantitative densitometric analysis of $\mathbf{u}$. w Invasion of GPR160 gene silenced or transfected cells. x Quantitative densitometric analysis of w. y Wound-healing assay in GPR160 gene silenced or transfected cells. Data presented are means \pm SEM obtained from three independent experiments; ${ }^{*} P<0.05$, ${ }^{* *} P<0.01$, and ${ }^{* * *} P<0.001$ using Student's $t$ test 
properties. Alterations in EMT hallmarks such as decreases in E-cadherin and ZO-1 and increases in N-cadherin, vimentin, snail, and fibronectin 1 are indicative of cell mobility. Here, we provided evidence to link the presence of GPR160 to cell mobility according to the fact that most patients with positive GPR160 protein staining exhibited lower $M$ scores.

In this study, there were 131 samples (53.7\%) examined for GPR160 mRNA and protein in parallel. Among them, 5/36 (13.9\%) of negative immunohistochemistry (IHC) samples were in agreement with that of fluorescence in situ hybridization (FISH), and 90/ 95 (94.8\%) of IHC-positive samples were verified by that of FISH, giving a total consistency rate of $72.5 \%$ resulting in a Kappa coefficient of $0.111(P=0.097)$ and Fisher's exact test $P$ value of 0.137. A high discordance was observed among $\mathrm{FISH}+/ \mathrm{IHC}-$ patients (Table S3), suggesting that the level of GPR160 transcription does not correlate with that of translation. Discordant protein and mRNA expression have been well documented for gastric cancer, breast cancer and lung adenocarcinoma. ${ }^{5}$ In our case, the observed discordance might have been caused by a number of factors such as GPR160 translational regulation, different sensitivity of the two detection systems, receptor degradation by proteinase during assaying, etc. Obviously, posttranscriptional regulation of GPR160 in prostate cancer development requires in-depth investigation to further validate this biomarker.

GPCRs, as cell surface proteins to react with different ligands and transmit signals into intracellular molecules, have been implicated in the formation of various tumors. The involvement of GPR160 in prostate cancer demonstrated by this histological study, including correlation with the Gleason score, PSA level, metastasis, and prior treatment, offers potential application of this novel biomarker in targeted therapy and post-surgical evaluation of tumor malignancy and prognosis.

\section{DATA AVAILABILITY}

The data sets used for the current study are available from the corresponding author upon reasonable request.

\section{ACKNOWLEDGEMENTS}

We are indebted to Qifeng Wang, Lijun Shao, Yingna $\mathrm{Xu}$, and Yanling Feng for technical assistance. This work was partially supported by grants from the National Natural Science Foundation of China 81872915 (M.W.W.), 81673470 (C.H.Z.), 81773792 (D.H.Y.), and 81973373 (D.H.Y.), the National Science \& Technology Major Project "Key New Drug Creation and Manufacturing Program" of China (2018ZX09735-001 to M.W.W. and 2018ZX09711002-002-005 to D.H.Y.), the National Key R\&D Program of China (2017YFC0114303 to B.D. and 2018YFA0507000 to M.W.W.), Strategic Priority Research Program of the Chinese Academy of Sciences XDA12020347 (M.W.W.), 2016 CAS Key Laboratory of Receptor Research External Collaboration Project (B.D.), the Natural Science Foundation of Shanghai Municipality 16ZR1406500 (D.B.), the Guide Project of Science and Technology Commission of Shanghai Municipality 17411963100 (B.D.), the Shanghai Cancer Research Charity Center Fund (B.D.) and Novo Nordisk-CAS Research Fund NNCAS-2017-1-CC (D.H.Y.).

\section{AUTHOR CONTRIBUTIONS}

M.W.W., B.D., and D.H.Y. designed research. W.J.G., J.Y.Z., Y.Z., C.H.Z., Y.J.Y., Z.T.C., and J.B.D. performed research, W.J.G., J.Y.Z., B.D., D.H.Y., and M.W.W. analyzed data. W.J.G., J.Y.Z., D.H.Y., B.D., and M.W.W. drafted, edited, and revised the manuscript. The funders had no role in study design, data collection, and analysis, decision to publish or manuscript preparation.

\section{ADDITIONAL INFORMATION}

Supplementary information The online version contains supplementary material available at https://doi.org/10.1038/s41392-021-00583-7.

Competing interests: The authors declare no competing interests.

Wanjing Guo ${ }^{1,2}$, Junyu Zhang ${ }^{3}$, Yan Zhou ${ }^{1}$, Caihong Zhou ${ }^{1}$, Yunjie Yang ${ }^{3}$, Zhaotong Cong ${ }^{4}$, Jibin Dong ${ }^{4}$, Dehua Yang ${ }^{1,2}$ Bo Dai ${ }^{3}$ and Ming-Wei Wang $\mathbb{D}^{1,2,4}$

${ }^{1}$ The National Center for Drug Screening and CAS Key Laboratory of Receptor Research, Shanghai Institute of Materia Medica, Chinese Academy of Sciences (CAS), Shanghai, China; ${ }^{2}$ University of Chinese Academy of Sciences, Beijing, China; ${ }^{3}$ Department of Urology, Fudan University Shanghai Cancer Center, Shanghai, China and ${ }^{4}$ School of Pharmacy, Fudan University, Shanghai, China These authors contributed equally: Wanjing Guo and Junyu Zhang. Correspondence: Dehua Yang (dhyang@simm.ac.cn) or Bo Dai (bodai1978@126.com) or Ming-Wei Wang (mwwang@simm. ac.cn)

\section{REFERENCES}

1. Ferlay, J. et al. Cancer incidence and mortality worldwide: sources, methods and major patterns in GLOBOCAN 2012. Int J. Cancer 136, E359-E386 (2015).

2. Yosten, G. L. et al. GPR160 de-orphanization reveals critical roles in neuropathic pain in rodents. J. Clin. Invest. 130, 2587-2592 (2020).

3. Zhou, C. et al. G protein-coupled receptor GPR160 is associated with apoptosis and cell cycle arrest of prostate cancer cells. Oncotarget 7, 12823-12839 (2016).

4. Chaffer, C. L., San Juan, B. P., Lim, E. \& Weinberg, R. A. EMT, cell plasticity and metastasis. Cancer Metastasis Rev. 35, 645-654 (2016).

5. Gopal, G., Shirley, S., Raja, U. M. \& Rajkumar, T. Endo-sulfatase Sulf-1 protein expression is down-regulated in gastric cancer. Asian Pac. J. Cancer Prev. 13, 641-646 (2012)

Open Access This article is licensed under a Creative Commons Attribution 4.0 International License, which permits use, sharing, adaptation, distribution and reproduction in any medium or format, as long as you give appropriate credit to the original author(s) and the source, provide a link to the Creative Commons license, and indicate if changes were made. The images or other third party material in this article are included in the article's Creative Commons license, unless indicated otherwise in a credit line to the material. If material is not included in the article's Creative Commons license and your intended use is not permitted by statutory regulation or exceeds the permitted use, you will need to obtain permission directly from the copyright holder. To view a copy of this license, visit http://creativecommons org/licenses/by/4.0/.

(c) The Author(s) 2021 